\title{
The Comparison of the Early Outcomes of Perimembranous Ventricular Septal Defect Closure by Two Different Methods: Transcatheter and Open- Heart Surgery in the Northwest of Iran
}

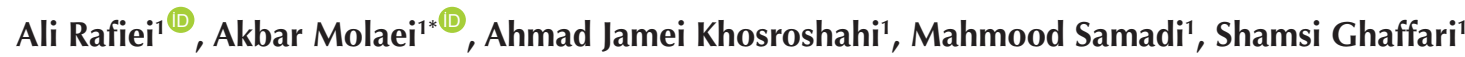

\begin{abstract}
Objectives: Ventricular septal defect (VSD) is the most common congenital heart disease (CHD) (40\%). Traditionally, VSDs have been repaired surgically; however, recently VSD closure by device has been developed. This study aimed to compare these two methods in terms of effectiveness, outcomes, and complications.

Materials and Methods: This study was conducted on VSD patients who were treated by either surgery or trans catheter technique in Madani Heart Center from March 2011 to March 2017. The required data regarding effectiveness, outcomes, and complications were recorded based on the patient's documents. The extracted data were analyzed by SPSS version 18.0.

Results: A total of 93 patients were enrolled (43 patients in transcatheter [A] and 50 patients in open-heart surgery [B] group) in this study. The gender distribution was approximately the same. The mean age of group A was 10.55 years while it was 8.8 years for group B. The duration of ICU and hospital stay, total hospitalization cost, and frequency of blood products transfusion were significantly lower in group A than in group B. However, postoperative pacemaker insertion, arrhythmias, thromboembolism, and mortality rates did not show any significant difference between the two groups. The success rates in group A and B were $97.7 \%$ and $96 \%$, respectively.

Conclusions: The results of our study demonstrated that open-heart surgery, due to its nature, required longer hospital and ICU stay, higher cost, and higher blood transfusion rate than transcatheter therapy, but it was associated with similar complications and success rates. Therefore, device closure in appropriately selected patients is recommended as an alternative to the surgical approach.
\end{abstract}

Keywords: Ventricular septal defect, Open-heart surgery, Transcatheter, Outcomes

\section{Introduction}

Ventricular septal defect (VSD) is the most common congenital heart disease (CHD) (40\%) with a prevalence of 3-3.5 per 1000 live births. Same as other CHDs, VSDs arise from earlier problems in the development of the heart, but often there is no clear cause. Genetics and environmental factors may play a role. VSDs may run in families and sometimes may occur with other genetic problems, such as Down syndrome. Maternal diseases such as diabetes mellitus and the use of drugs such as antiepileptics and tricyclic antidepressants may be associated. The location of this defect and its size determine the symptoms and the course of the disease. Those defects which lead to significant left to right shunt and have clinical symptoms should be treated before irreversible complications occur. The most common type is perimembranous VSD which accounts for about $80 \%$ of cases (2).

The natural course of isolated interventricular septal defects indicates that some of them are closed during follow-up or become smaller (4) and some of them are at high risk of pulmonary hypertension during infancy which need to be surgically repaired as soon as possible. Others have complications such as aortic insufficiency, infective endocarditis, or sub-valvar pulmonary stenosis $(2,3)$.

Traditionally, interventricular defects have been surgically closed using special patches of pericardium or synthetic material with excellent results $(1,5)$.

In recent years, a new method has been advised for closing some of these defects without open-heart surgery through central vessels by transcatheter procedure and an occluder (5). This procedure was performed on children and adults $(4,6,7)$ for both muscular (8) and perimembranous VSDs (9-12). Due to vascular access limitation in infants and young children for percutaneous transcatheter device implantation, the surgical closure (13) or periventricular device implantation (14) was recommended. In this method, since the patient is treated without open-heart surgery and cardiopulmonary bypass, it is necessary to perform a statistical and analytical study 


\section{Key Message}

- Percutaneous VSD closure by device is a safe and effective alternate for surgery.

- This procedure should be performed in equipped centers with trained and experienced staff.

in order to compare the results of treatment and probable complications of this method with surgery and draw conclusions.

In this study, we decided to compare open heart surgery and transcatheter procedure in terms of the results, complications, outcomes, and cost of hospitalization in order to compare and analyze the advantages and disadvantages in our hospital.

Therapeutic methods include either open-heart surgery $(1,5)$ or transcatheter device closure (1).

\section{Materials and Methods}

This cross-sectional descriptive study was conducted on patients with VSD who were treated by either open heart surgery or transcatheter technique in Madani Heart Center from March 2011 to March 2017.

The variables such as success rate, incidence of bleeding requiring blood transfusion, arrhythmia, cardiac block, postoperative infection, mortality, hospital and ICU stay duration, and hospitalization cost were evaluated.

Required data were extracted from patients' medical records and echocardiography reports produced after one year. It should be noted that all patients treated with transcatheter procedure were exposed to radiation during coronary angiography and fluoroscopy and all patients treated with open-heart surgery underwent cardiopulmonary bypass. The collected data were analyzed by descriptive statistics (frequency, percentage, mean, and standard deviation) using independent samples $t$ test, chi-square test, and Fisher exact test. Additionally, Kolmogorov-Smirnov test was used for assessing the normality of data distribution by SPSS version 18.0. In this study, $P$ value $\leq 0.05$ was considered statistically significant.

\section{Results}

This study was performed on 93 patients with isolated perimembraneous VSD. A total of 43 patients underwent transcatheter procedure (group A) and 50 patients underwent open-heart surgery (group B). Variables including age, weight, and gender of the two groups were matched. The study found a significantly shorter length of stay in ICU and hospital and lower hospitalization cost in group A (Table 1).

All patients in group B underwent general anesthesia, but only $2(3.8 \%)$ patients in group A were necessarily intubated in this study $(P<0.001)$. In the surgery group, 4 (8\%) patients required insertion of temporary pacemaker due to transient atrioventricular block $(P=0.058)$. In this study, 2 (4.7\%) patients in group A had left bundle branch block after the procedure that was resolved by corticosteroid therapy (prednisolone $2 \mathrm{mg} / \mathrm{kg}$ for two weeks that was tapered in additional two weeks) after 3-4 weeks.

None of the patients needed permanent pacemaker implantation. There was more need for blood transfusion in group $B$.

In terms of postoperative complications such as arrhythmia, thromboembolism, residual shunt requiring surgical intervention, and mortality rate, there were no significant differences between the two groups (Table 2).

Generally, 3 (7\%) patients in group A showed mild tricuspid valve regurgitation (TR) after device implantation. The device embolization occurred in one patient in the descending aorta and the device was extracted by snare and re-implanted.

\section{Discussion}

VSD is the most common CHD. Those defects which lead to significant left to right shunt and have clinical symptoms should be treated before irreversible complications occur (1). The procedure of choice for VSDs repair is surgical closure by patch with excellent results $(1,5)$. In recent years, the percutaneous transcatheter closure by occluders has been introduced (1). This procedure was performed on children and adults $(3,7,8)$ for both muscular $(9)$ and PM VSDs (10-13).

A total of 93 patients with VSD were enrolled in this study. Fifty patients underwent open-heart surgery and 43 patients underwent transcatheter VSD closure. In this study, age, weight, and gender of the two groups were matched. The study found that the length of stay in ICU and hospital was significantly shorter and the hospital costs were significantly lower in the transcatheter group.

In the studies conducted by Oses et al (15) and Chen et al (16), a significantly shorter duration of surgery and mechanical ventilation, lower cost of hospitalization, and shorter duration of ICU and hospital stay were observed in the transcatheter group compared to the open-heart surgery group, the same as our study.

Table 1. Comparison of the Quantitative Parameters of the Patients in Two Groups (Mean \pm SD)

\begin{tabular}{lccc}
\hline Parameter & Group (A) & Group (B) & P Value \\
\hline Age (year) & $10.55 \pm 5.70$ & $8.80 \pm 5.07$ & 0.199 \\
$\begin{array}{l}\text { Gender } \\
\quad \text { Female }\end{array}$ & & \\
$\quad$ Male & $18(41.9 \%)$ & $25(50 \%)$ & 0.432 \\
Weight (kg) & $35.27 \pm 21.5$ & $27.57 \pm 17.02$ & 0.057 \\
ICU stays (day) & $1.00 \pm 0.37$ & $4.86 \pm 1.76$ & $<0.001$ \\
Hospital stay (day) & $3.84 \pm 0.63$ & $12.04 \pm 3.86$ & $<0.001$ \\
$\begin{array}{l}\text { Cost of treatment } \\
(\times 10 \text { million IRR) }\end{array}$ & $6.558 \pm 48.68$ & $10.653 \pm 62.01$ & 0.001 \\
\hline
\end{tabular}

ICU, Intensive Care Unit.

Group A: Transcatheter group. Group B: Open heart surgery group. 
Table 2. Comparison of Success Rate and Complications in Two Groups

\begin{tabular}{lccc}
\hline & Group (A) & Group (B) & $\boldsymbol{P}$ Value \\
\hline Success rate & & & \\
Successful & $41(95.3 \%)$ & $49(98 \%)$ & \\
Failure & $2(4.7 \%)$ & $1(2 \%)$ & 0.432 \\
Post-operative arrhythmias & $6(14 \%)$ & $6(12 \%)$ & 0.779 \\
Blood products requirement & $2(4.7 \%)$ & $45(90 \%)$ & $<0.001$ \\
Residual shunt & $1(2.35 \%)$ & $2(4 \%)$ & 0.469 \\
Mortality & 0 & 0 & 0 \\
Pacemaker insertions & $0(0 \%)$ & $4(8.0 \%)$ & 0.058 \\
Thromboembolism & 0 & 0 & 0 \\
\hline
\end{tabular}

Our study showed that the need for blood products was significantly lower in the transcatheter group $(P<0.001)$ but the need for post-operative pacemaker $(P=0.058)$, post-operative arrhythmia $(P=0.779)$, incidence of postoperative thromboembolism, residual shunt requiring re-intervention $(P=0.649)$, and mortality rate were not significantly different between the two groups. In the study conducted by Oses et al (15), no significant difference was found between the two groups in the incidence of residual shunt and the need for a pacemaker, which is similar to our study.

In a study by Carminati et al (17), the residual shunt was detected in $4 \%$ of patients after transcatheter closure. Transient left bundle branch block was observed in two patients and transient first degree AV block was observed in one patient, the same as our study. Among patients with PM VSD, complete AV block was observed in 3 patients within the first 48 hours. One patient needed a pacemaker. In this study, major complications were observed in the transcatheter group, and it was found that $6.47 \%$ had severe arrhythmia, $16.85 \%$ of patients had TR, and $17 \%$ needed transfusion of blood and blood products. In 11.2\% of patients, pleural effusion and pericardial effusion were seen, all of which were treated. Additionally, 17.58\% of patients undergoing surgery had severe arrhythmia, $17.5 \%$ of patients had aortic valve insufficiency, and $3.34 \%$ of patients had TR. In our study, there was not any atrioventricular block or pleural or pericardial effusion in the transcatheter group but transient left bundle branch block and TR were seen in 2 and 3 patients, respectively.

In the study conducted by Szkutnik et al (18), displacement of the device was not seen in any of the patients. The incidence of arrhythmia was higher in the transcatheter group $(P=0.08)$. In the surgical group, complete atrio-ventricular block and TR were observed in $11.1 \%$ and $33 \%$ of patients, respectively. In our study, device embolization occurred in one patient in the descending aorta and the device was extracted by snare and re-implanted. In studies by Nguyen et al (19) and Liu et al (20), the incidence of complications after transcatheter repair was $1.7 \%$, including $\mathrm{CHB}$ in $0.7 \%$ and device embolization in $1 \%$ of the patients.

In a study by Yang et al (21), it was reported that major complications including mortality, need for re- operation due to complications, and need for pacemaker implantation were observed in one patient in both groups and no differences were observed between the two groups. Moreover, there was no significant difference in the incidence of $\mathrm{CHB}$ and valvar insufficiency between the two groups.

The more complex operation, the higher the complication rate is. However, comparing these two methods, it was observed that most of the complications in the two groups were approximately the same and major complications were not significantly different between the two groups. The rate of complications such as pain, surgical site infection, and complications associated with surgical incision may be significantly higher in the surgical group, but these complications were not assessed in these studies.

In our study, the therapeutic success rate was $96 \%$ in the surgical group and it was $97.7 \%$ in the transcatheter group $(P=0.649)$. In the study conducted by Oses et al (15), it was reported that $11.1 \%$ of patients in the transcatheter group and $23.5 \%$ in the SG group had residual shunt after surgery but the shunt was not observed at follow-up. In the study conducted by Carmineti et al (17), it was reported that the success rate of VSD closure by transcatheter technique was $97.5 \%$. This procedure was not performed in 3 patients $(2.45 \%)$ due to the impossibility of sheath insertion in 1 patient, the onset of complete AV block during manipulation of catheter in 1 patient, and the presence of aortic valve prolapse in 1 patient.

In the study by Carminati et al (17), the early success rate of transcatheter technique was $61.4 \%$ and reached up to $100 \%$ within three months. In the study by Szkutnik et al (18), the success rate of both groups was $88.88 \%$ and no significant difference was observed in the success rate of the two groups. In the study conducted by Nguyen et al (19), the success rate of the transcatheter method was $95.6 \%$, and the success rates of defect closure within 24 hours and 6 months, and 2 years were 89.5\%, 91.5\%, $99.3 \%$, respectively. In a study by Liu et al (20), the success rate of transcatheter technique was $99.36 \%$ and the success rate of surgical technique was $98.93 \%$, indicating no significant difference between these two methods. In the study by Yang et al (21), it was also observed that the success rate of transcatheter method was $99 \%$ and it was $99.3 \%$ for surgical method, indicating no significant difference between these two methods.

The success rate in almost all studies was the same (above 98\%) in both groups, indicating the high efficacy of both methods, which was clearly seen in our study.

Although at early stages, the incidence of residual shunt may be higher in the transcatheter group than in the surgical group, the success rate is almost the same in the end.

In our study, no in-hospital death was recorded. This result is the same for studies conducted by Zuo et al (12), Oses et al (15), Carminati et al (17), and Nguyen et al (19). 
However, in the study conducted by Zhuang et al (14), it was reported that in-hospital mortality rate was $5.65 \%$ and the main causes were pulmonary hypertension crisis and arrhythmia. Studies have shown that the surgical death rate beyond infancy due to VSD repair is close to zero.

\section{Conclusions}

Due to the minimally invasive nature of transcatheter approach for VSD closure, length of hospitalization and ICU stays is shorter, and medical costs are lower compared to the surgical approach.

The results of this study showed that open-heart surgery due to its nature requires mechanical ventilation, prolonged anesthesia, and ICU and hospital stay, higher cost, and more blood transfusion compared to transcatheter method. However, the complications and success rates of these two therapeutic methods are almost equal. Therefore, transcatheter method in appropriately selected patients in well-equipped centers with experienced and trained staff can be an appropriate alternative to openheart surgery.

\section{Limitations of Study}

The small sample size of studied patients can be regarded as the limitation of the study; therefore, the confirmation of the results requires further research with a larger population. Another limitation was the failure to investigate mortality and out-of-hospital mortality rate.

\section{Authors' Contribution}

AM: study conception and design; AR: data collection; AJK: analysis and interpretation of results; MS and SG: draft manuscript preparation. All authors reviewed the results and approved the final version of the manuscript.

\section{Conflict of Interests}

Authors have no conflict of interest.

\section{Ethical Issues}

Ethics Committee of Tabriz University of Medical Sciences approved this study (No: IR.TBZMED.REC.1397.838).

\section{Financial Support}

This study was supported by Cardiovascular Center of Tabriz University of Medical Sciences.

\section{Acknowledgments}

The authors would like to thank all of the patients who participated indirectly in this study and thank Cardiovascular Research Center of Tabriz University of Medical Sciences for providing financial support for this project.

\section{References}

1. Allen HD, Driscoll DJ, Shaddy RE, Feltes TF. Moss \& Adams' Heart Disease in Infants, Children, and Adolescents: Including the Fetus and Young Adult. Lippincott Williams \& Wilkins; 2013.

2. Behjati-Ardakani M, Soltani MH, Dehghani-Firoozabadi R. The Clinical Course of Ventricular Septal Defect. J Mazandaran Univ Med Sci. 2011;21(85):159-164. [Persian].

3. Libby P, Bonow RO, Mann DL, Zipes DP. Braunwald's Heart
Disease: A Textbook of Cardiovascular Medicine, 2-Volume Set. Elsevier Health Sciences; 2007.

4. Kouchoukos NT, Blackstone EH, Hanley FL, Kirklin JK. Kirklin/Barratt-Boyes Cardiac Surgery E-Book. Elsevier Health Sciences; 2012.

5. Mehta AV, Goenka S, Chidambaram B, Hamati F. Natural history of isolated ventricular septal defect in the first five years of life. Tenn Med. 2000;93(4):136-138.

6. Warnes CA, Williams RG, Bashore TM, et al. ACC/AHA 2008 guidelines for the management of adults with congenital heart disease: a report of the American College of Cardiology/ American Heart Association Task Force on Practice Guidelines (Writing Committee to Develop Guidelines on the Management of Adults with Congenital Heart Disease). Developed in Collaboration with the American Society of Echocardiography, Heart Rhythm Society, International Society for Adult Congenital Heart Disease, Society for Cardiovascular Angiography and Interventions, and Society of Thoracic Surgeons. J Am Coll Cardiol. 2008;52(23):e143-e263. doi:10.1016/j.jacc.2008.10.001

7. Baumgartner $\mathrm{H}$, Bonhoeffer $\mathrm{P}$, De Groot NM, et al. ESC Guidelines for the management of grown-up congenital heart disease (new version 2010). Eur Heart J. 2010;31(23):29152957. doi:10.1093/eurheartj/ehq249

8. Holzer R, Balzer D, Cao QL, Lock K, Hijazi ZM. Device closure of muscular ventricular septal defects using the Amplatzer muscular ventricular septal defect occluder: immediate and mid-term results of a U.S. registry. J Am Coll Cardiol. 2004;43(7):1257-1263. doi:10.1016/j.jacc.2003.10.047

9. Hijazi ZM, Hakim F, Haweleh AA, et al. Catheter closure of perimembranous ventricular septal defects using the new Amplatzer membranous VSD occluder: initial clinical experience. Catheter Cardiovasc Interv. 2002;56(4):508-515. doi:10.1002/ccd.10292

10. Yang J, Yang L, Yu S, et al. Transcatheter versus surgical closure of perimembranous ventricular septal defects in children: a randomized controlled trial. J Am Coll Cardiol. 2014;63(12):1159-1168. doi:10.1016/j.jacc.2014.01.008

11. Saurav A, Kaushik M, Mahesh Alla V, et al. Comparison of percutaneous device closure versus surgical closure of peri-membranous ventricular septal defects: a systematic review and meta-analysis. Catheter Cardiovasc Interv. 2015;86(6):1048-1056. doi:10.1002/ccd.26097

12. Zuo J, Xie J, Yi W, et al. Results of transcatheter closure of perimembranous ventricular septal defect. Am J Cardiol. 2010;106(7):1034-1037. doi:10.1016/j.amjcard.2010.05.040

13. Zhuang J, Zhang J, Cen J, Chen X, Chen J, Chen W. [Surgical treatment of ventricular septal defect in infants: report of 230 cases]. Zhonghua Wai Ke Za Zhi. 1999;37(4):241-242.

14. Molaei A, Afrasiabi A, Bilejani E, Samadi M. Perventricular muscular ventricular septal defect (VSD) closure under epicardial echocardiography guidance: a case report. J Tehran Heart Cent. 2017;12(3):138-141.

15. Oses $\mathrm{P}$, Hugues $\mathrm{N}$, Dahdah $\mathrm{N}$, et al. Treatment of isolated ventricular septal defects in children: Amplatzer versus surgical closure. Ann Thorac Surg. 2010;90(5):1593-1598. doi:10.1016/j.athoracsur.2010.06.088

16. Chen Q, Cao H, Zhang GC, Chen LW, Li QZ, Qiu ZH. Closure of perimembranous ventricular septal defects with intraoperative device technique: another safe alternative to surgical repair. Thorac Cardiovasc Surg. 2013;61(4):293-299. doi:10.1055/s-0032-1311532

17. Carminati M, Butera G, Chessa M, Drago M, Negura D, Piazza L. Transcatheter closure of congenital ventricular septal defect with Amplatzer septal occluders. Am J Cardiol. 2005;96(12A):52L-58L. doi:10.1016/j.amjcard.2005.09.068 
18. Szkutnik M, Kusa J, Białkowski J. Percutaneous closure of perimembranous ventricular septal defects with Amplatzer occluders--a single centre experience. Kardiol Pol. 2008;66(9):941-947.

19. Nguyen HL, Phan QT, Doan DD, et al. Percutaneous closure of perimembranous ventricular septal defect using patent ductus arteriosus occluders. PLoS One. 2018;13(11):e0206535. doi:10.1371/journal.pone.0206535
20. Liu S, Chen F, Ding X, et al. Comparison of results and economic analysis of surgical and transcatheter closure of perimembranous ventricular septal defect. Eur J Cardiothorac Surg. 2012;42(6):e157-162. doi:10.1093/ejcts/ezs519

21. Yang J, Yang L, Wan Y, et al. Transcatheter device closure of perimembranous ventricular septal defects: mid-term outcomes. Eur Heart J. 2010;31(18):2238-2245. doi:10.1093/ eurheartj/ehq240

Copyright $\left({ }^{\circ} 2022\right.$ The Author(s); This is an open-access article distributed under the terms of the Creative Commons Attribution License (http://creativecommons.org/licenses/by/4.0), which permits unrestricted use, distribution, and reproduction in any medium, provided the original work is properly cited. 